\title{
SYNTHESIS OF FLUOROPHOSPHATE GLASSES WITH LOW MELTING TEMPERATURES
}

\author{
J. Leissner, K. Sebastian, H. Roggendorf and H. Schmidt \\ Fraunhofer Institut für Silicatforschung \\ Neunerplatz 2, D-8700 Würzburg, FRG
}

\begin{abstract}
Fluorophosphate glasses can combine low melting temperatures with a good chemical durability. In order to vary optical properties while retaining other features some new compositions were synthesized and some of their properties were investigated. The glasses were melted at $450{ }^{\circ} \mathrm{C}$ by using Sno, $\mathrm{PbF}_{2}, \mathrm{SnF}_{2}, \mathrm{NH}_{4} \mathrm{H}_{2} \mathrm{PO}_{4}$ and $\mathrm{NH}_{4} \mathrm{PF}_{6}$ as raw materials. Metal fluorides like $\mathrm{ZrF}_{4}$ and $\mathrm{ZnF}_{2}$ were introduced, too. The $\mathrm{T}_{\mathrm{g}}$ ranges between 87 and $141^{\circ} \mathrm{C}$. Preliminary results concerning the transmission spectra in the UV, visible and medium IR range, the indices of refraction, and the densities are reported.
\end{abstract}

\section{INTRODUCTION}

Interest in fluorophosphate glasses developed from the combination of low melting temperatures, good chemical durability and desirable optical properties, such as a relatively high transmission in a broad spectral range, a high Abbe number and a wide range of the index of refraction. A carefully melted fluorophosphate glass exhibits ultraviolet transparency comparable to that of glasses based on $\mathrm{BeF}_{2}$ and fused silica [1].

In spite of a significant interest in these glasses, few systematic studies on glass forming compositions and their properties have been reported. Therefore the objective of the present work 
was to investigate glasses of various compositions in the $\mathrm{PbF}_{2}-$ $\mathrm{SnF}_{2}-\mathrm{SnO}-\mathrm{P}_{2} \mathrm{O}_{5}$ system and the effect of the addition of $\mathrm{ZnF}_{2}$ or $\mathrm{ZrF}_{4}$. The initial starting point was one of Tick's glasses [2] (glass I in table 1), which shows a very good resistance to water attack and has a low glass transition temperature $\mathrm{T}_{\mathrm{g}}$.

\section{BATCH COMPOSITION}

The basic batch composition (glass I) is covered by the system Sno-SnF $2-\mathrm{PbF}_{2}-\mathrm{P}_{2} \mathrm{O}_{5}$ (the batch mixtures are listed in table 1). The first step was to simply add $\mathrm{ZrF}_{4}$ (glass II) to the original formula of Tick [2]. The next variation was to reduce the oxygen content by replacing sno by the corresponding equimolar amount of $\mathrm{SnF}_{2}$ and by adding increasing amounts of $\mathrm{ZnF}_{2}$ (glasses III - V). A further increase of the fluorine content was tried by introducing $\mathrm{NH}_{4} \mathrm{PF}_{6}$ into the batch, the concentration of this compound ranged between 2.73 and 21.97 wt. of (glasses VI-VIII).

Table 1: Batch composition of the investigated glasses in wt $\frac{8}{8}$

\begin{tabular}{lcccccccr} 
Batch & \multicolumn{7}{c}{ Glass } \\
& I & II & III & IV & V & VI & VII & VIII \\
$\mathrm{SnF}_{2}$ & 30.70 & 30.27 & 58.08 & 57.28 & 56.51 & 57.28 & 48.62 & 45.93 \\
$\mathrm{SnO}$ & 26.43 & 26.04 & - & - & - & - & - & - \\
$\mathrm{PbF}_{2}$ & 6.75 & 6.65 & 6.38 & 6.30 & 6.20 & 6.30 & 5.34 & 5.05 \\
$\mathrm{NH}_{4} \mathrm{H}_{2} \mathrm{PO}_{4}$ & 36.12 & 35.60 & 34.16 & 33.69 & 33.24 & 33.69 & 28.60 & 27.05 \\
$\mathrm{NH}_{4} \mathrm{PF}_{6}$ & - & - & - & - & - & 2.73 & 17.44 & 21.97 \\
$\mathrm{ZrF}_{4}$ & - & 1.44 & - & - & - & - & - & - \\
$\mathrm{ZnF}_{2}$ & - & - & 1.38 & 2.73 & 4.05 & - & - & -
\end{tabular}

\section{EXPERIMENTAL PROCEDURE}

The glasses were melted in a $50 \mathrm{~cm}^{3}$ vitreous carbon crucible using analytical grade raw materials. The crucible was placed into a muffle furnace at $450^{\circ} \mathrm{C}$ and heated for about 45 minutes. Due to the strong evaporation it was not possible to use a covered crucible. Clear fluid melts were obtained, which could readily be cast at room temperature into a brass mould. 
The obtained transparent glasses were analyzed by $x$-ray diffraction ( $\mathrm{Cu} \mathrm{K \alpha}$; glass plates of $4 \times 5 \mathrm{~cm}^{2}$ in size, thickness about $0.3 \mathrm{~cm}$ ). The composition was determined by chemical analysis of $\mathrm{F}$. Two glasses (glass II and VIII) were also analyzed with respect to $\mathrm{P}, \mathrm{Pb}, \mathrm{Sn}$, and $\mathrm{Zr} ; \mathrm{NH}_{\mathrm{x}}$ was analyzed qualitatively in glasses II and VIII; the oxygen content was not analyzed. The glass transformation temperature $\mathrm{T}_{\mathrm{g}}$ was measured by differential scanning calorimetry (DSC) or differential thermal analysis (DTA) and the optical transmission by UV-, VIS-, and IR-spectroscopy of cast glass plates or powdered glass dispersed in KBr-pellets (IR). The index of refraction was determined by the immersion method and the density by applying Archimedes principle. A rough information of the chemical durability was obtained by leaching glass fragments in water at room temperature with subsequent balancing and optical control of the surface roughness.

\section{RESULTS}

\subsection{GLASS FORMATION AND STRUCTURE}

All batches yielded in clear glasses. Glass VIII was quite unstable and crystallized during storage in air. The X-ray diffraction diagramms of six glasses are shown in figure 1. Diffuse bands typical of vitreous materials can be seen. The intensity of the band at about $26^{\circ}(2 \theta)$ differs a bit. Glasses II - VIII seem to have a higher degree of structural order than glass $I$.

\subsection{COMPOSITION}

Due to volatilisation the chemical analysis (tables 2 and 3 ) showed a severe loss of $F$ of about $50-70 \%$ with respect to the relative batch content. The highest $\mathrm{F}$-content was achieved with batch VIII. Also $\mathrm{Pb}$ and $\mathrm{P}$ seem to form rather volatile components. The relatively high loss of $\mathrm{Zr}$ cannot be explained at the moment. $\mathrm{NH}_{4}{ }^{+}$was qualitatively detected in the leaching solutions of both analyzed glasses. This indicates that $\mathrm{NH}_{\mathrm{x}}$-groups are still incorporated in the glass. 


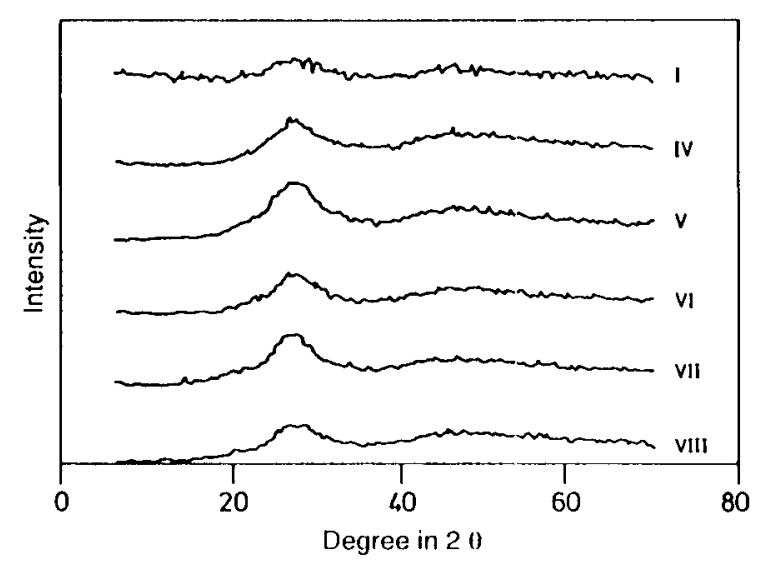

Figure 1: X-ray-diffractograms of glasses I and IV to VIII. The compositions are listed in table 1 .

Table 2: Calculated elemental batch composition and chemical analysis of glass II and VI.LI in wt .

\begin{tabular}{lrrrr} 
Glass & \multicolumn{2}{c}{ II } & \multicolumn{2}{c}{ VIII } \\
& & & & \\
& & & & \\
$\mathrm{F}$ & 8.94 & 4.4 & 27.12 & 11.8 \\
$\mathrm{P}$ & 9.59 & 9.2 & 11.42 & 11.9 \\
$\mathrm{~Pb}$ & 5.63 & 5.2 & 4.26 & 5.6 \\
$\mathrm{Sn}$ & 45.69 & 55.3 & 34.68 & 52.4 \\
$\mathrm{Zr}$ & 0.75 & 0.3 & - & -
\end{tabular}

\subsection{TRANSFORMATION TEMPERATURE $T$ AIJD DENSITY}

The $\mathrm{T}_{\mathrm{g}}$ (table 3 ) values vary between $87^{\circ} \mathrm{C}$ (glass VIII) and $141^{\circ} \mathrm{C}$ (glass II) and decrease with the increasing fluorine content. The considerable increase of $\mathrm{NH}_{4} \mathrm{PF}_{6}$ in the batch (glasses VI - VIII) results in a significant decrease of $\mathrm{T}_{\mathrm{g}}$ of about $30 \mathrm{~K}$. The glasses I and III - V have similar densities of about 3.91 $3.96 \mathrm{~g} \cdot \mathrm{cm}^{-3}$, whereas the $\mathrm{ZrF}_{4}$-containing $\mathrm{glass}$ II has the highest density of $4.05 \mathrm{~g} \cdot \mathrm{cm}^{-3}$. The addition of $\mathrm{NH}_{4} \mathrm{PF}_{6}$ leads to a significant decrease of the density (table 3 ). 


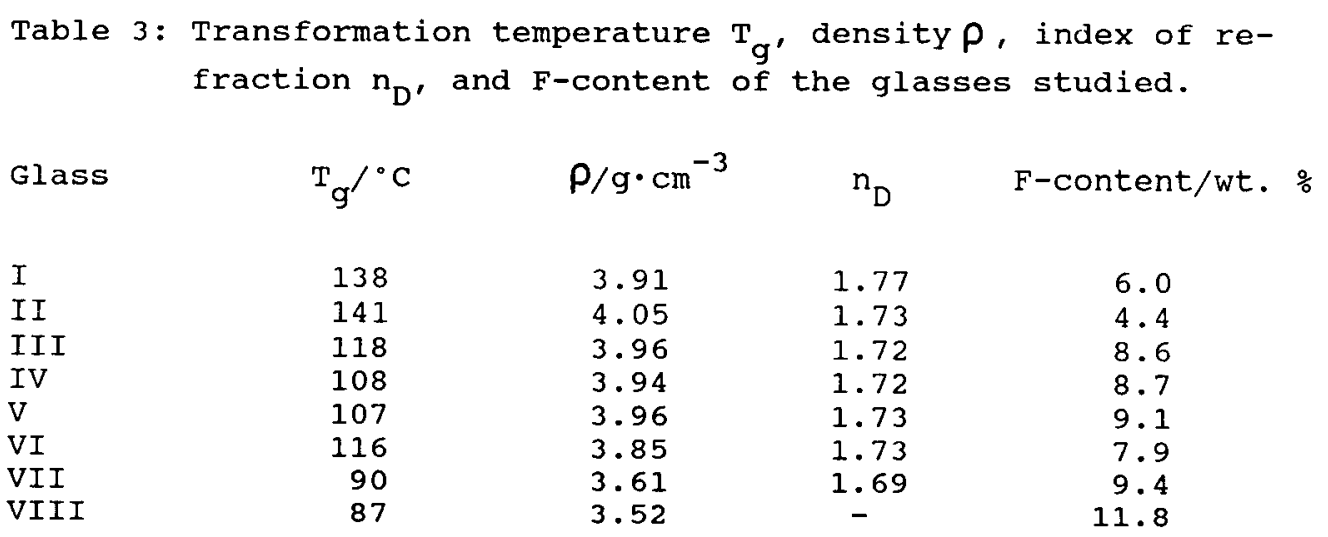

\subsection{OPTICAL TRANSMISSION AND INDEX OF REFRACTION}

The prepared glasses have a good transparency in the visible and the near infrared. The UV cut off is at about $320 \mathrm{~nm}$ and is independant on the glass composition. In the normal IR range the glasses are transparent between 4000 and $1200 \mathrm{~cm}^{-1}$. This indicates the absence of oH-groups. At about $1400-1600 \mathrm{~cm}^{-1}$ an absorption band is observed typical of $\mathrm{NH}$-vibrations.

No remarkable differences were observed for any glasses. Figure 2 shows a transmission spectrum of glass VIII, typical of all the examined glasses.

The $\mathrm{n}_{\mathrm{D}}$ values cannot be exactly correlated to the density (table 3), but they decrease with increasing F-content.

\subsection{CHEMICAL DURABILITY}

Glasses I and II showed no evident change after 1 hour in water but the surface of glasses III to VIII (tin oxide free batches) was strongly damaged after 1 hour. Similar tests in organic solvents like ethanol, acetone and dichloromethane showed no significant attack for any glasses.

\section{CONCLUSIONS}

On the basis of the glass system $\mathrm{PbF}_{2}-\mathrm{SnO}-\mathrm{SnF}_{2}-\mathrm{NH}_{4} \mathrm{H}_{2} \mathrm{PO}_{4}$ new glass compositions were realised using a melting procedure. The F-content was increased up to $11.8 \mathrm{wt}$. $\%$

The most important influence on the properties of the glasses results from the equimolar substitution of $\mathrm{Sno}$ by $\mathrm{SnF}_{2}$ in the batch 


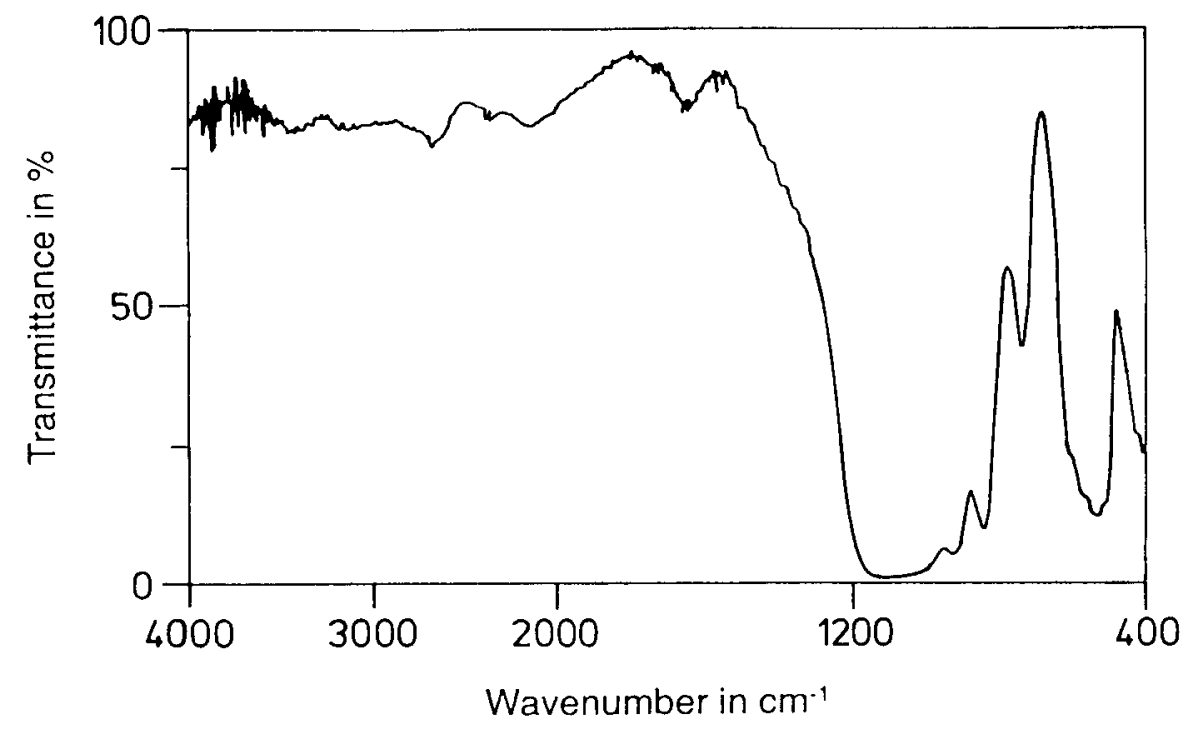

Figure 2: Transmission spectrum of glass IV in the IR range.

of glasses III - VIII. By this means the F-content is increased and the amount of metal-oxygen-bonds is probably reduced resulting in a decrease of $\mathrm{T}_{\mathrm{g}^{\prime}}$ density, and chemical durability. By further addition of fluorine by $\mathrm{NH}_{4} \mathrm{PF}_{6}$ the $\mathrm{T}_{\mathrm{g}}$ decreases below $90^{\circ} \mathrm{C}$. The incorporated $\mathrm{NH}_{\mathrm{x}}$-groups are leading to the formation of $\mathrm{NH}_{4}^{+}$-ions in the leaching solutions. The structure of the incorporated nitrogen has not been investigated, but the content is considered to be responsible at least partially for the properties of these glasses.

6. REFERENCES

1) Cook L. and Mader K.: J. Am. Ceram. Soc. 1982, 45, 597.

2) Tick P. A.: Phys. and Chem. of Glasses 1984, 25, 149. 